\section{Colistin-Resistant Klebsiella pneumoniae: Report of a Cluster of 24 Cases from a New Oncology Center in Eastern India}

To the Editor-Early detection and identification of antimicrobial resistance is important with regard to clinical and infection control challenges. ${ }^{1}$ Colistin is a broad-spectrum antibiotic used in the treatment of drug-resistant gram-negative bacterial infections. It is especially useful for treatment of carbapenemase-producing gram-negative bacilli. Resistance to this very important antibiotic represents a serious challenge to the therapeutic armamentarium. In this article, we describe a series of 24 cases of colistin-resistant Klebsiella (CRK) infection or colonization from a new oncology center in the eastern part of India. These 24 cases of CRK infection were documented between June 15, 2013, and February 3, 2014. This hospital is a tertiary care center for patients with cancer and also performs bone marrow transplantation. CRK was detected by Vitek 2 compact (microbroth dilution using nephelometric methods). Each Vitek 2 result was also confirmed by E-test methods (bioMérieux), and a minimum inhibitory concentration of $2 \mu \mathrm{g} / \mathrm{mL}$ or greater was defined as indicating resistance, in accordance with the British Society for Antimicrobial Chemotherapy. Surveillance cultures for antibiotic-resistant bacteria in stool and other specimens were performed according to a method based on a modification to the technique described by Landman et al. ${ }^{2}$ The mean age of the patients infected or colonized was 41.1 years (mean ages of male and female patients were 43.2 and 36.9 years, respectively). Male-to-female patient ratio was $2: 1$. The patients were being treated in different departments ( 5 in the Gastrointestinal Surgery Department, 2 each in the Head and Neck Surgery Department and Urological Surgery Department, 1 each in the Gynecology Department and Medical Oncology Department, and 13 in the Clinical Hematology Department). The number of patients with blood cultures positive for CRK was 10; other specimens from which CRK was isolated included respiratory specimens, aspirates, and pus. The number of patients previously receiving colistin before isolation of CRK was $15(62.5 \%)$ of 24 . In total, 18 patients survived and 6 died (all cause mortality, 25\%). The mean duration of hospital stay after detection of CRK was 17.9 days. Two patients received outpatient care only. Stool surveillance cultures were obtained for 5 patients before detection of CRK $(20.8 \%)$. The clinical infection types noted included sepsis in 15 patients, pneumonia in 4 patients, urosepsis in 1 patient, and soft-tissue infection in 1 patient. Three patients had asymptomatic colonization. Twenty $(83.3 \%)$ of 24 patients had cases that came under the category of healthcare-associated infection with culture positivity 48 hours or more after hospital admission. Ten (41.7\%) of 24 patients had previous carbapenamase-producing Klebsiella isolated from 1 or more specimens before detection of CRK. Among other antibiotics, the susceptibility profile of tigecycline was noteworthy, with 14 isolates showing intermediate susceptibility and 5 showing susceptibility. Few isolates showed intermediate susceptibility or susceptibility to amikacin, gentamicin, chloramphenicol, minocycline, fosfomycin, and cotrimoxazole. Patients were treated on the basis of antibiotic susceptibility test results.

Infection control measures were applied after detection of CRK. Barrier precautions were universally applied. These included universal precautions, appropriate use of personal protective equipment, enhanced and supervised housekeeping and cleaning activities, emphasis on hand hygiene, electronic flagging of the biohazard symbol on the hospital information system screen, a physical flagging of affected patients with a "safety first" board near the bed, e-mail communications of CRK results to relevant clinical and supporting staff groups, and staff education. ${ }^{3}$

There is a high prevalence of antibiotic resistance in this geographical area. The prevalences of extended-spectrum $\beta$ lactamase and carbapenemase producers in bloodstream infections in the same period were approximately $70 \%$ and $39 \%$, respectively. There is consequently high use of meropenem and colistin in this hospital of 167 beds. During the study period, the defined daily doses of meropenem and colistin ranged from 243 to 428 and from 87 to 216 , respectively. Colistin resistance in Klebsiella species can either arise de novo because of colistin-induced antibiotic pressure or be horizontally transmitted because of breakdown of infection control measures. ${ }^{4-6}$ The clonality of these isolates is yet to be established (laboratory-based molecular typing has been planed). Typing of CRK isolates can be done by both phenotypic and genotypic methods. However, DNA-based typing methods, such as restriction fragment-length polymorphism, pulsed-field gel electrophoresisis, and multilocus sequence typing are superior. It is not for the first time that CRK is being reported. Case series have been documented from Italy, the United States, Hungary, and Greece. ${ }^{4-10}$ However, such reports are rare, although we suspect underreporting of cases. One of the challenges faced in the developing world is lack of isolation facilities in adequate numbers and the lack of adequate awareness, which makes infection control a priority. The situation is complicated further by poor hygiene and sanitation and inadequate disposal of biomedical and general wastes in the developing world, along with over-the-counter availability of broad-spectrum antibiotics. There is also a lack of discipline in antibiotic prescribing, and antibiotic stewardship programs are not well developed in many hospitals. CRK is symptomatic of a serious problem that is due to a complex interaction of various factors that arise both in the healthcare setting and in the community. Extreme care is required to contain this pathogen, which represents a grave threat to human health. We also suggest surveillance culture of patients for antibiotic-resistant bacteria both at the time 
of hospital admission and at weekly intervals to detect asymptomatic carriage. ${ }^{10}$

\section{ACKNOWLEDGMENTS}

Potential conflicts of interest. All authors report no conflicts of interest relevant to this article. All authors submitted the ICMJE Form for Disclosure of Potential Conflicts of Interest, and the conflicts that the editors consider relevant to this article are disclosed here.

Gaurav Goel, MD; ${ }^{1}$ Lalawmpuia Hmar, $\mathrm{MD}^{1}$ Maitrayee Sarkar De, BSc, MSc; Sanjay Bhattacharya, MD, DNB, DipRCPath, FRCPath; ${ }^{1}$ Mammen Chandy, MD, FRACP, FRCPA ${ }^{1}$

Affiliation: 1. Tata Medical Center, Kolkata, India.

Address correspondence to Sanjay Bhattacharya, MD, DNB, DipRCPath, FRCPath, Consultant Microbiologist, Tata Medical Center, South Lab, 14 Major Arterial Road (E-W), New Town, Rajarhat, Kolkata 700 156, India (drsanjay1970@hotmail.com).

Infect Control Hosp Epidemiol 2014;35(8):1076-1077

(C) 2014 by The Society for Healthcare Epidemiology of America. All rights reserved. 0899-823X/2014/3508-0027\$15.00. DOI: $10.1086 / 677170$

\section{REFERENCES}

1. Bhattacharya S. Early diagnosis of resistant pathogens: how can it improve antimicrobial treatment? Virulence 2013;4:172-184.

2. Landman D, Salvani JK, Bratu S, Quale J. Evaluation of techniques for detection of carbapenem-resistant Klebsiella pneumoniae in stool surveillance cultures. J Clin Microbiol 2005;43:5639_ 5641.

3. Bhattacharya S, Das D, Bhalchandra R, Goel G. Patient isolation in the high-prevalence setting: challenges with regard to multidrug-resistant gram-negative bacilli. Infect Control Hosp Epidemiol 2013;34:650-651.

4. Tóth A, Damjanova I, Puskás E, et al. Emergence of a colistinresistant KPC-2-producing Klebsiella pneumoniae ST258 clone in Hungary. Eur J Clin Microbiol Infect Dis 2010;29:765-769.

5. Kontopoulou K, Protonotariou E, Vasilakos K, et al. Hospital outbreak caused by Klebsiella pneumoniae-producing KPC-2 $\beta$ lactamase resistant to colistin. J Hosp Infect 2010;76:70-73.

6. Bogdanovich T, Adams-Haduch JM, Tian GB, et al. Colistinresistant, Klebsiella pneumoniae carbapenemase (KPC)-producing Klebsiella pneumoniae belonging to the international epidemic clone ST258. Clin Infect Dis 2011;53:373-376.

7. Jernigan MG, Press EG, Nguyen MH, Clancy CJ, Shields RK. The combination of doripenem and colistin is bactericidal and synergistic against colistin-resistant, carbapenemase-producing Klebsiella pneumoniae. Antimicrob Agents Chemother 2012;56: 3395-3398.

8. Tascini C, Tagliaferri E, Giani T, et al. Synergistic activity of colistin plus rifampin against colistin-resistant KPC-producing Klebsiella pneumoniae. Antimicrob Agents Chemother 2013;57: 3990-3993.

9. Hong JH, Clancy CJ, Cheng S, et al. Characterization of porin expression in Klebsiella pneumoniae carbapenemase (KPC)-producing K. pneumoniae identifies isolates most susceptible to the combination of colistin and carbapenems. Antimicrob Agents Chemother 2013;57:2147-2153.

10. Bhattacharya S. Is screening patients for antibiotic-resistant bacteria justified in the Indian context? Indian J Med Microbiol 2011; 29:213-217.

\section{Fatal Outbreak of Polyclonal Candidemia in a Neonatal Intensive Care Unit}

To the Editor-Candida species are the third most common cause of late-onset sepsis in neonatal intensive care units (NICUs), accounting for 9\%-13\% of cases, with an increasing trend compared with previous surveillance studies. ${ }^{1}$ The mortality associated with invasive candidiasis (IC) is consistently high, with death rates reaching $40 \%-50 \%{ }^{2}$ The most frequently isolated species is Candida albicans, although nonalbicans species have emerged as important opportunistic pathogens. ${ }^{3}$ Early colonization may come from the mother (vertical transmission) or from the environment or hospital staff (horizontal transmission). ${ }^{4}$ Risk factors for colonization and posterior infection are prematurity, very low birth weight (VLBW), male sex, vaginal delivery, indwelling catheters, total parenteral nutrition, broad-spectrum antibiotics, ventilatory support, gastrointestinal disorders, previous documented history of bloodstream infection, and abdominal surgery. ${ }^{2,5,6}$ Clinical diagnosis is difficult to establish, because IC resembles sepsis syndrome and has a variety of clinical manifestations, such as respiratory insufficiency, feeding intolerance, abdominal distention, temperature instability, and lethargy. Therefore, the definition of IC is a positive culture of a sterile body fluid sample, which can have a sensitivity of only $29 \%{ }^{3,7}$

On January 24, 2013, the State Committee of Hospital Infection Control was notified of the occurrence of 5 newborn deaths of unknown etiology in an NICU of a large public hospital in the Brazilian Amazon within a period of 4 days. On the same day, an investigation was conducted to confirm the outbreak, identify risk factors for infection, identify the microbiological agent involved, and propose recommendations to prevent new infections. Twenty-nine newborns were evaluated in the NICU between December 31, 2012, and March 19, 2013, from admission of the first suspected case patient up to 1 month without the registry of new cases. The NICU consisted of 14 individual nurseries, which, because of the suspected outbreak, had new admissions suspended until laboratory results were available and control measures were in place (a 2-week period). Blood specimens for culture were collected from all newborns and sent to the reference laboratory. Isolates were recovered with a BD Bactec system (Becton Dickinson), and any fungal growth was then cultured on Sabouraud dextrose agar media. Some of the isolates were analyzed by pulsed-field gel electrophoresis (PFGE). We also evaluated all infection control procedures adopted in the unit: 